\title{
Online Appendix: Adverse Selection and Auction Design for Internet Display Advertising
}

\author{
By Nick Arnosti and Marissa Beck and Paul Milgrom
}

Note that for any allocation rule $z$, the quantities $V_{B}(z), V_{P}(z)$ and $V(z)$ depend implicitly on the number of bidders and the distributions of advertiser values. At times, we study the variation in the performance of an allocation rule $z$, as a function of an underlying parameter $\theta$ (such as the number of bidders or the distribution from which their values are drawn). In some cases, we make the dependence on $\theta$ explicit by writing $V(z ; \theta)$. Throughout the appendix, we use the letter $\mu$ to refer to the brand advertiser's expected match value $E\left[M_{0}\right]$.

\section{PROOF OF PROPOSITION 1:}

We must show that second price auctions cannot guarantee $\left(\frac{1}{2}+\epsilon\right) V(\mathrm{OMN})$, for any $\epsilon>0$. Fix $N \geq 2$ and $\epsilon>0$. To do so, we assume that $M_{i}$ are iid draws from a power law distribution with parameter $a$, and maintain the identity $\gamma \mu=\gamma E\left[M_{0}\right]=(1+\epsilon) E\left[M_{(1)}\right]$. As $a \downarrow 1$, it becomes possible to capture nearly all of the value from performance advertisers by allocating to them a vanishingly small fraction of impressions. Thus,

$$
\frac{V(\mathrm{OMN})}{E[C] E\left[M_{(1)}\right]}=\frac{E\left[\max \left(\gamma \mu, M_{(1)}\right)\right]}{E\left[M_{(1)}\right]} \rightarrow \frac{\gamma \mu+E\left[M_{(1)}\right]}{E\left[M_{(1)}\right]}=2+\epsilon .
$$

Choose $a$ sufficiently close to one such that $V(\mathrm{OMN})>2 E\left[M_{(1)}\right] E[C]$. Let $C$ be drawn from a power law distribution with parameter $a^{\prime}$. By Lemma 1, if $a^{\prime}$ is sufficiently close to one, then $\sup _{b} V\left(\mathrm{SP}_{b}\right)=\gamma \mu E[C]=(1+\epsilon) E\left[M_{(1)}\right] E[C]$. It follows that $\sup _{b} V\left(\mathrm{SP}_{b}\right) / V(\mathrm{OMN})<\frac{1+\epsilon}{2}$.

LEMMA 1: Suppose that $M_{(1)} \sim F$, which has density $f$ on $[0, \infty)$, and that $E\left[M_{(1)}^{1+\epsilon}\right]<\infty$ for some $\epsilon>0$. Fix $\gamma \mu=\gamma E\left[M_{0}\right]>E\left[M_{(1)}\right]$. Suppose that $C \in[1, \infty)$ has density $g(c)=a^{\prime} c^{-a^{\prime}-1}$. Then there exists $\delta>0$ such that if $a^{\prime}<1+\delta$,

$$
\sup _{b} V\left(\mathrm{SP}_{b}\right)=\gamma \mu E[C]
$$

\section{PROOF OF LEMMA 1:}

Note that this is equivalent to showing that if $a$ is sufficiently small, then the brand advertiser wants to increase its bid without bound (i.e. always win). 
We see that

$$
\begin{aligned}
V\left(\mathrm{SP}_{b}\right) & =\gamma E\left[C \mu \mathbf{1}_{C M_{(1)} \leq b}\right]+E\left[C M_{(1)} \mathbf{1}_{C M_{(1)}>b}\right] \\
& =\gamma \mu \int_{1}^{\infty} c F(b / c) g(c) d c+\int_{1}^{\infty} \int_{m=b / c}^{\infty} c m f(m) g(c) d m d c
\end{aligned}
$$

From this, if follows that

$$
\begin{aligned}
\frac{d}{d b} V\left(\mathrm{SP}_{b}\right) & =\int_{1}^{\infty}(\gamma \mu-b / c) f(b / c) g(c) d c . \\
& =a^{\prime} b^{-a^{\prime}} \int_{0}^{b}(\gamma \mu-u) f(u) u^{a^{\prime}-1} d u,
\end{aligned}
$$

where we have performed the change of variables $u=b / c$ and used the fact that $g(c)=a^{\prime} c^{-a^{\prime}-1}$.

We will show that for all $a^{\prime}$ sufficiently close to one, the above expression is non-negative for all $b$, implying that it is optimal for the brand advertiser to win all impressions. Viewed as a function of $b$, the integral $\int_{0}^{b}(\gamma \mu-u) f(u) u^{a^{\prime}-1} d u$ is (weakly) increasing on $[0, \gamma \mu]$ and (weakly) decreasing thereafter. Thus, it is enough to show that when $a^{\prime}$ is sufficiently small, $\int_{0}^{\infty}(\gamma \mu-u) f(u) u^{a^{\prime}-1} d u>0$.

Because $E\left[M_{(1)}^{1+\epsilon}\right]<\infty$ for some $\epsilon>0$, we may apply the dominated convergence theorem to see that as $a^{\prime} \downarrow 1$,

$$
\int_{0}^{\infty}(\gamma \mu-u) f(u) u^{a^{\prime}-1} d u \rightarrow \int_{0}^{\infty}(\gamma \mu-u) f(u) d u=\gamma \mu-E\left[M_{(1)}\right]>0 .
$$

\section{PROOF OF PROPOSITION 2:}

For a reminder of the theory of sufficient statistics, see Theory of Point Estimation by Lehmann and Casella. We begin by establishing that $\left(N, X_{(N)}\right)$ is a sufficient statistic for $C$ (and thus for $X_{0}$ ). Given $C$ and $N$, the conditional density of $X$ is

$$
f(X ; C, N)=a^{N} C^{a N} \mathbf{1}_{X_{(N)} \geq C} \cdot\left(\prod_{i=1}^{N} X_{i}\right)^{-a-1}
$$

so $X_{(N)}$ is a sufficient statistic for $C$.

We now show that $E\left[C \mid N, X_{(N)}\right]$ is non-decreasing in $X_{(N)}$. The notation that follows assumes that $C$ follows an atomless distribution with density $g$, although the argument can be extended to the case where the distribution of $C$ has atoms.

The conditional density of $C$, given $\left(N, X_{(N)}\right)$, is proportional to $g(c) c^{a N+1}$ on 
$\left[1, X_{(N)}\right]$. Thus,

$$
E\left[C \mid N, X_{(N)}=x\right]=\frac{\int_{0}^{x} g(c) c^{a N+2} d c}{\int_{0}^{x} g(c) c^{a N+1} d c} .
$$

We wish to prove that this expression is non-decreasing in $x$. Its derivative with respect to $x$ is

$$
\frac{g(x) x^{a N+2} \int_{0}^{x} g(c) c^{a N+1} d c-g(x) x^{a N+1} \int_{0}^{x} g(c) c^{a N+2} d c}{\left(\int_{0}^{x} g(c) c^{a N+1} d c\right)^{2}},
$$

which can be rewritten as

$$
\frac{g(x) x^{a N+1}}{\int_{0}^{x} g(c) c^{a N+1} d c}\left(x-E\left[C \mid N, X_{(N)}=x\right]\right) .
$$

The first term is clearly non-negative, as is the second term (because $M_{(N)} \geq 1$ and $\left.X_{(N)}=C M_{(N)} \geq C\right)$.

We now turn to the second point in the proposition, which states that under OPT, $E\left[z_{0}(X) \mid C, N\right]$ is decreasing in $C$ whenever $C$ follows a power law distribution.

We begin with a series of claims that hold whenever the match values follow a power law distribution (regardless of the distribution of $C$ ). First, we claim that

$$
E\left[z_{0}(X) \mid N, X_{(N)}\right]=E\left[z_{0}(X) \mid C, N, X_{(N)}\right]
$$

that is, given the values of $N$ and $X_{(N)}$, the value of $C$ does not affect the probability that the impression is awarded to the brand advertiser. To see this, recall that under OPT,

$$
z_{0}(X)=\mathbf{1}_{X_{(1)} \leq \gamma E\left[X_{0} \mid X\right]}=\mathbf{1}_{\frac{X_{(1)}}{X_{(N)}} \leq \gamma E\left[M_{0}\right] \cdot \frac{E\left[C \mid N, X_{(N)}\right]}{X_{(N)}},}
$$

where the first equality holds by definition and the second makes use of the fact that $\left(N, X_{(N)}\right)$ is a sufficient statistic for $C$. Clearly, the distribution of $X_{(1)} / X_{(N)}=M_{(1)} / M_{(N)}$ is independent from $C$, implying that (2) holds. From this and the definition of conditional expectation, it follows that

$$
\begin{aligned}
E\left[z_{0}(X) \mid C, N\right] & =E\left[E\left[z_{0}(X) \mid C, N, X_{(N)}\right] \mid C, N\right] . \\
& =E\left[E\left[z_{0}(X) \mid N, X_{(N)}\right] \mid C, N\right] .
\end{aligned}
$$

Because the conditional distribution of $X_{(N)}$ given $C, N$ is stochastically increasing in $C$, to show that $E\left[z_{0}(X) \mid C, N\right]$ is decreasing in $C$, it suffices to show that 
$E\left[z_{0}(X) \mid N, X_{(N)}\right]$ is decreasing in $X_{(N)}$. From (3), we see that

$$
E\left[z_{0}(X) \mid N, X_{(N)}\right]=P\left(\frac{X_{(1)}}{X_{(N)}} \leq \gamma E\left[M_{0}\right] \cdot \frac{E\left[C \mid N, X_{(N)}\right]}{X_{(N)}} \mid N, X_{(N)}\right) .
$$

Because $X_{(1)} / X_{(N)}$ is ancillary for $C$, and $X_{(N)}$ is sufficient for $C$, Basu's theorem implies that $X_{(1)} / X_{(N)}$ is conditionally independent from $X_{(N)}$, given $N$. Therefore, in order to show that the quantity $E\left[z_{0}(X) \mid N, X_{(N)}\right]$ is decreasing in $X_{(N)}$, it is enough to show that the ratio $E\left[C \mid N, X_{(N)}=x\right] / x$ is decreasing in $x$.

Here, for the first time, we invoke the assumption that $C$ follows a power law distribution - that is, that $g(x)=b x^{-b-1}$ on $[1, \infty)$ for some $b>1$. Define $\beta=a N-b+2$. By (1), we have that

$$
\frac{1}{x} E\left[C \mid N, X_{(N)}=x\right]=\left\{\begin{array}{ll}
\frac{\log (x)}{x-1} & \beta=0 \\
\frac{x-1}{x \log (x)} & \beta=1 \\
\frac{\beta-1}{\beta} \cdot \frac{x^{\beta}-1}{x^{\beta}-x} & \beta \notin\{0,1\}
\end{array} .\right.
$$

In what follows, we assume $\beta \notin\{0,1\}$; similar arguments establish monotonicity of the expressions corresponding to $\beta=0$ and $\beta=1$. Differentiating with respect to $x$, we see that

$$
\begin{aligned}
\frac{d}{d x} \frac{1}{x} E\left[C \mid N, X_{(N)}=x\right] & =\frac{\beta-1}{\beta}\left(x^{\beta}-x\right)^{-2}\left(\left(x^{\beta}-x\right)\left(\beta x^{\beta-1}\right)-\left(x^{\beta}-1\right)\left(\beta x^{\beta-1}-1\right)\right) . \\
& =\frac{\beta-1}{\beta}\left(x^{\beta}-x\right)^{-2}\left(\beta x^{\beta-1}+x^{\beta}-\beta x^{\beta}-1\right) .
\end{aligned}
$$

We must show that for $x>1$, this expression is negative. Its sign is determined by the sign of

$$
\frac{\beta-1}{\beta}\left(\beta x^{\beta-1}+x^{\beta}-\beta x^{\beta}-1\right),
$$

which takes the value zero at $x=1$. Thus, it is enough to show that this quantity is non-increasing in $x$ for $x \geq 1$. Differentiating, we get

$$
\frac{\beta-1}{\beta} \frac{d}{d x}\left(\beta x^{\beta-1}+x^{\beta}-\beta x^{\beta}-1\right)=(\beta-1)^{2} x^{\beta-2}(1-x) \leq 0 .
$$

\section{PROOF OF THEOREM 1:}

By inspection, any MSB auction is strategy-proof, deterministic, anonymous, false-name proof and adverse selection free. Conversely, it is well-known that any strategy-proof deterministic and anonymous mechanism is characterized by a "threshold price" function $h$ such, for any competing bids $x_{-i}$, bidder $i$ wins if and only if its bid exceeds its threshold price $h\left(x_{-i}\right)$ and conditional on winning, $i$ pays this threshold price. Any such mechanism also has the property that only 
the top performance bidder can win, which requires that $h\left(x_{-i}\right) \geq \max \left\{x_{-i}\right\}$.

We claim that if the mechanism is false-name proof, then $h\left(x_{-i}\right)=h\left(\max \left\{x_{-i}\right\}\right)$. Suppose that there exists exists $x_{-i}$ such that $h\left(x_{-i}\right) \neq h\left(\max \left\{x_{-i}\right\}\right)$, and examine the incentives when there are two bidders, one with value exceeding $h\left(x_{-i}\right)$ and the other with value $\max \left\{x_{-i}\right\}$. If $h\left(x_{-i}\right)<h\left(\max \left\{x_{-i}\right\}\right)$, then the first bidder can reduce its price by submitting the remaining bids in the profile $x_{-i}$, so the mechanism is not winner false-name proof. If $h\left(x_{-i}\right)>h\left(\max \left\{x_{-i}\right\}\right)$, then the losing bidder can raise the winner's price by submitting the remaining bids in the profile $x_{-i}$, so the mechanism is not loser false-name proof.

Next, we show that if the mechanism is adverse selection free, $h$ must be homogeneous of degree one. For suppose not. Then there exists $c \in \mathbb{R}_{+}, n \geq 2$, and $x_{-i} \in \mathbb{R}_{+}^{n-1}$ such that (without loss of generality) $h\left(m_{-i}\right)<h\left(c m_{-i}\right) / c$. Fix $m_{i} \in\left(h\left(m_{-i}\right), h\left(c m_{-i}\right) / c\right)$. Suppose that $C \in\{1, c\}$ with $P(C=1) \in(0,1)$, that $P\left(M_{-i}=m_{-i}\right)=1$, and that $P\left(M_{i}=m_{i}\right)=1$. We show that $z_{0}(C M)=\mathbf{1}_{\{C=c\}}$, proving that the auction associated with $h$ is not adverse-selection free.

When $C=1, z_{i}(C M)=z_{i}(m)=\mathbf{1}_{\left\{m_{i}>h\left(m_{-i}\right)\right\}}=1$, so $z_{0}(C M)=0$. When $C=$ $c, z_{i}(C M)=z_{i}(\mathrm{~cm})=\mathbf{1}_{\left\{\mathrm{cm}_{i}>h\left(\mathrm{~cm}_{-i}\right)\right\}}=0$. Because only the top performance bidder (bidder $i$ ) can win the auction, this implies that $z_{0}(C M)=1$.

Thus, for any mechanism that is deterministic, strategy proof, false-name proof, and adverse-selection free, there is a threshold price function $h$ that is homogeneous of degree one and depends only on its maximum argument: $h\left(\max \left\{x_{-i}\right\}\right)=$ $\alpha \max \left\{x_{-i}\right\}$ for some $\alpha$. The fact that $h\left(x_{-i}\right) \geq \max \left\{x_{-i}\right\}$ implies $\alpha \geq 1$.

\section{PROOF OF PROPOSITION 3:}

Note that $\alpha=1$ and $\alpha=\infty$ describe the cases where the brand advertiser never wins or always wins, so $V\left(\mathrm{MSB}_{1}\right)=E\left[X_{(1)}\right]$ and $V\left(\mathrm{MSB}_{\infty}\right)=\gamma E\left[X_{0}\right]$. Furthermore,

$$
\begin{aligned}
V(\mathrm{OMN}) & =E\left[\max \left(\gamma C E\left[M_{0}\right], C M_{(1)}\right)\right] \\
& \leq \gamma E\left[X_{0}\right]+E\left[X_{(1)}\right] \\
& \leq 2 \cdot \max \left(\gamma E\left[X_{0}\right], E\left[X_{(1)}\right]\right)
\end{aligned}
$$

which proves the first claim.

For the second claim, we let $C$ be distributed according to $G(x)=1-x^{-b}$ on $[1, \infty)$. Fix $N \geq 2$, and suppose the $M_{i}$ are iid draws from $F(x)=x^{\beta / N}$ on $[0,1]$. Straightforward calculations reveal that if we define $\hat{g}$ to be the conditional density of $C$ given performance values $X$, then

$$
\hat{g}(c)=\frac{(\beta+b)}{\max \left(X_{(1)}, 1\right)}\left(\frac{c}{\max \left(X_{(1)}, 1\right)}\right)^{-\beta-b-1} \text { on }\left[\max \left(X_{(1)}, 1\right), \infty\right) .
$$

In other words, given $X, C$ is distributed as a power law random variable with 
parameter $(b+\beta)$, conditioned on being greater than $\max \left(X_{(1)}, 1\right)$. It follows that

$$
E\left[X_{0} \mid X\right]=E[\mu C \mid X]=\frac{\beta+b}{\beta+b-1} \mu \max \left(X_{(1)}, 1\right) .
$$

If $\gamma \mu=\frac{\beta+b-1}{\beta+b}$, then $\gamma E[\mu C \mid X]=\max \left(X_{(1)}, 1\right)$, so it is optimal to always award the impression to the brand advertiser. This generates a total value of

$$
V(\mathrm{OPT})=\gamma E[\mu C]=\frac{b}{b-1} \frac{\beta+b-1}{\beta+b} .
$$

Meanwhile, straightforward calculations reveal that the first-best solution generates value

$$
V(\mathrm{OMN})=E[C] E\left[\max \left(\gamma \mu, M_{(1)}\right)\right]=\frac{b}{b-1}\left(\frac{\beta}{1+\beta}+\frac{(\gamma \mu)^{1+\beta}}{1+\beta}\right) .
$$

As $b \rightarrow 1, \gamma \mu \rightarrow \frac{\beta}{\beta+1}$. From this, we see that

$$
\lim _{b \rightarrow 1} V(\mathrm{OMN} ; b) / V(\mathrm{OPT} ; b)=1+\frac{\beta^{\beta}}{(1+\beta)^{1+\beta}} .
$$

As $\beta \rightarrow 0$, this tends to 2 , implying that even the optimal mechanism cannot guarantee more than $1 / 2$ of the value generated by the first-best solution.

We now turn to the proof of Theorem 2. Throughout this section, we assume that $N$ is deterministically equal to $n \geq 2$, and that the $M_{i}$ are iid draws from a distribution with density $f$ and $\operatorname{cdf} F$. We use the letter $\mu$ to represent the brand advertisers expected match value $E\left[M_{0}\right]$. Rather than specifying $\mu$ directly, we choose an alternative parameterization by letting $\lambda \in[0,1]$ be the probability that the brand advertiser receives the impression under the first-best solution, and defining $\mu(\lambda, n)$ by

$$
\lambda=F(\mu(\lambda, n))^{n},
$$

Thus, $\mu(\lambda, n)$ gives the brand advertiser's expected value, as a function of the number of bidders $n$ and the fraction of impressions $\lambda$ won by the brand advertiser under the first-best allocation (throughout, we fix the distribution $F$ of each performance match value). For notational simplicity, we treat the case where $\gamma=1$. Other values of $\gamma$ follow identically, as changing $\gamma$ is effectively equivalent to rescaling the brand advertiser's average match value $\mu$.

We begin with a technical lemma, which allows us to compute $V_{P}(\mathrm{OMN})$, given $\lambda, n$, and the function $\mu(\lambda, n)$.

LEMMA 2: Suppose that $P(N=n)=1$ and that the $M_{i}$ are iid draws from a distribution with density $f$ and $c d f F$. Let $\lambda=P\left(M_{(1)} \leq E\left[M_{0}\right]\right)$, and define the 
function $\mu$ as in (4). Then

$$
V_{P}(\mathrm{OMN})=E[C] \int_{\lambda}^{1} \mu(x, n) d x .
$$

PROOF OF LEMMA 2:

Differentiating the identity $F(\mu(\lambda, n))^{n}=\lambda$, we obtain

$$
n F(\mu(\lambda, n))^{n-1} f(\mu(\lambda, n)) \frac{d}{d \lambda} \mu(\lambda, n)=1 .
$$

Therefore,

$$
\begin{aligned}
\frac{d}{d \lambda} V_{P}(\mathrm{OMN}) & =\frac{d}{d \lambda} E[C] E\left[M_{(1)} \mathbf{1}_{M_{(1)}>\mu(\lambda, n)}\right] \\
& =\frac{d}{d \lambda} \int_{\mu(\lambda, n)}^{\infty} x n F(x)^{n-1} f(x) d x \\
& =-\mu(\lambda, n) n F(\mu(\lambda, n))^{n-1} f(\mu(\lambda, n)) \frac{d}{d \lambda} \mu(\lambda, n) \\
& =-\mu(\lambda, n),
\end{aligned}
$$

where the final line follows from application of (5). The Lemma follows immediately.

Our proof of Theorem 2 references the gamma function $\Gamma$, defined by

$$
\Gamma(s)=\int_{0}^{\infty} x^{s-1} e^{-x} d x .
$$

We make use of the following facts.

FACT 1 (Power Law Distribution): Suppose that $\left\{M_{i}\right\}_{i=1}^{n}$ are IID draws from a power law distribution with parameter a, i.e. $P\left(M_{i} \leq x\right)=1-x^{-a}=F(x)$ for $x \in[1, \infty)$. Let $M_{(j)}$ be the $j^{\text {th }}$ order statistic of the $M_{i}$. Then

1) For any $r \geq 1, E\left[M_{i} \mid M_{i}>r\right]=r E\left[M_{i}\right]$.

2) $M_{(1)} / M_{(n)}, M_{(2)} / M_{(n)}, \ldots, M_{(n-1)} / M_{(n)}$ are independent from $M_{(n)}$ and are distributed as the order statistics of $M_{1}, \ldots, M_{n-1}$.

3) $E\left[M_{(1)}\right]=\Gamma(1-1 / a) \Gamma(n+1) / \Gamma(n+1-1 / a)$.

FACT 2 (Gamma Function):

1) For any $s>0, \Gamma(s+1)=s \Gamma(s)$.

2) For any $s>0, \lim _{n \rightarrow \infty}\left(\frac{n^{-s} \Gamma(n+1)}{\Gamma(n+1-s)}\right)=1$. 
LEMMA 3: If match values are independent draws from a power law distribution with parameter $a$, then for any $\alpha \geq 1$,

$$
V_{P}\left(\mathrm{MSB}_{\alpha}\right)=\alpha^{1-a} E\left[X_{(1)}\right]
$$

PROOF OF LEMMA 3:

$$
\begin{aligned}
V_{P}\left(\mathrm{MSB}_{\alpha}\right) & =E\left[X_{(2)} \frac{M_{(1)}}{M_{(2)}} \mathbf{1}_{\frac{M_{(1)}}{M_{(2)}}>\alpha}\right] \\
& =E\left[X_{(2)}\right] E\left[\frac{M_{(1)}}{M_{(2)}} \mathbf{1}_{M_{(1)}}\right] \\
& =E\left[X_{(2)}\right] E\left[\frac{M_{(1)}}{M_{(2)}}\right] \alpha P\left(\frac{M_{(1)}}{M_{(2)}}>\alpha\right) \\
& =E\left[X_{(2)} \frac{M_{(1)}}{M_{(2)}}\right] \alpha P\left(\frac{M_{(1)}}{M_{(2)}}>\alpha\right) \\
& =E\left[X_{(1)}\right] \alpha^{1-a}
\end{aligned}
$$

The first line uses the fact that $X_{(1)} / X_{(2)}=M_{(1)} / M_{(2)}$. The second and fourth lines use the independence of $M_{(2)}$ and $M_{(1)} / M_{(2)}$ established by Fact 1.2. The third and final lines use the fact that $M_{(1)} / M_{(2)}$ follows a power law distribution; the third line also applies Fact 1.1.

\section{PROOF OF THEOREM 2:}

Both OMN and MSB have allocation rules that are independent of $C$, so it is clear that the distribution of $C$ does not matter. For simplicity, in this proof we take $C$ to be identically one. This leaves us with three parameters of interest: the number of performance bidders $n$, the average value of the brand advertiser $\mu=E\left[M_{0}\right]$, and the weight of the power law tail, $a$. As above, we define $\lambda$ to be probability that the brand advertiser wins the impression under OMN, and use $\mu(\lambda, n)$ to refer to the brand value implied by the given values of $\lambda$ and $n$ (for fixed $a)$, so $\lambda=P\left(M_{(1)} \leq \mu(\lambda, n)\right)$.

The omniscient benchmark achieves total surplus given by

$$
\begin{aligned}
V(\mathrm{OMN}) & =V_{B}(\mathrm{OMN})+V_{P}(\mathrm{OMN}) \\
& =\lambda \mu(\lambda, n)+\int_{\lambda}^{1} \mu(x, n) d x .
\end{aligned}
$$


Meanwhile, for any $\alpha \geq 1$,

$$
\begin{aligned}
V\left(\mathrm{MSB}_{\alpha}\right) & =V_{B}\left(\mathrm{MSB}_{\alpha}\right)+V_{P}\left(\mathrm{MSB}_{\alpha}\right) \\
& =P\left(M_{(1)} \leq \alpha M_{(2)}\right) \mu(\lambda, n)+V_{P}\left(\mathrm{MSB}_{\alpha}\right) \\
& =\left(1-\alpha^{-a}\right) \mu(\lambda, n)+\alpha^{1-a} E\left[M_{(1)}\right]
\end{aligned}
$$

where the final line follows from Fact 1.2 and Lemma 3.

We choose the MSB parameter $\alpha$ such that the brand advertiser is awarded the impression with probability $\lambda$. In other words, we select $\alpha$ such that $1-\alpha^{-a}=\lambda$. Because both allocation rules deliver a representative sample of impressions to the brand advertiser, the first statement in Theorem 2 follows immediately. In other words, our choice of $\alpha$ ensures that $V_{B}(\mathrm{OMN})=V_{B}\left(\mathrm{MSB}_{\alpha}\right)$.

Of course, the value of impressions allocated to performance advertisers will be lower under MSB than under OMN. We establish in Lemma 4 that for fixed $\lambda$ and $a$, the ratio $V_{P}\left(\mathrm{MSB}_{\alpha}\right) / V_{P}(\mathrm{OMN})$ is decreasing in $n$. Applying Lemma 2, we see that

$$
\begin{aligned}
\lim _{n \rightarrow \infty} n^{-1 / a} V_{P}(\mathrm{OMN} ; n, \mu(\lambda, n)) & =\lim _{n \rightarrow \infty} n^{-1 / a} \int_{\lambda}^{1}\left(1-x^{1 / n}\right)^{-1 / a} d x \\
& =\int_{\lambda}^{1} \log (1 / x)^{-1 / a} d x
\end{aligned}
$$

(9)

By Lemma 3 and Facts 1.3 and 2.2, we see that

$$
\lim _{n \rightarrow \infty} n^{-1 / a} V_{P}\left(\mathrm{MSB}_{\alpha} ; n, \mu(\lambda, n)\right)=\lim _{n \rightarrow \infty} n^{-1 / a} \alpha^{1-a} E\left[M_{(1)}\right]=\alpha^{1-a} \Gamma(1-1 / a) .
$$

Lemma 5 establishes that the ratio $V_{P}\left(\mathrm{MSB}_{\alpha}\right) / V_{P}(\mathrm{OMN})$ worsens as $\lambda \rightarrow 1$. Taking $\lambda \rightarrow 1$ and applying L'Hospital's rule, we see that

$$
\begin{aligned}
\lim _{\lambda \rightarrow 1} \frac{(1-\lambda)^{1-1 / a} \Gamma(1-1 / a)}{\int_{\lambda}^{1} \log (1 / x)^{-1 / a} d x} & =(1-1 / a) \Gamma(1-1 / a) \lim _{\lambda \rightarrow 1} \frac{(1-\lambda)^{-1 / a}}{\log (1 / \lambda)^{-1 / a}} \\
& =\Gamma(2-1 / a) .
\end{aligned}
$$

where the final line follows from the identity $\Gamma(s+1)=s \Gamma(s)$ and the fact that $\lim _{\lambda \rightarrow 1}(1-\lambda) / \log (1 / \lambda)=1$. Because $a>1$, we have $2-1 / a \in(1,2)$. The minimum of the gamma function over the interval $(1,2)$ exceeds 0.885 , completing the proof of the second claim in Theorem 2.

We now turn our attention to the third claim. We show in Lemma 6 that $V\left(\mathrm{MSB}_{\alpha}\right) / V(\mathrm{OMN})$ is decreasing in $n$. We compute that

$$
\lim _{n \rightarrow \infty} n^{-1 / a} \mu(\lambda, n)=\log (1 / \lambda)^{-1 / a} .
$$


Combining this with (6), (7), (8) and (9), we conclude that

$$
\begin{gathered}
\lim _{n \rightarrow \infty} n^{-1 / a} V\left(\mathrm{MSB}_{\alpha} ; n, \mu(\lambda, n)\right)=\lambda \log (1 / \lambda)^{-1 / a}+\Gamma(1-1 / a)(1-\lambda)^{1-1 / a} . \\
\lim _{n \rightarrow \infty} n^{-1 / a} V(\mathrm{OMN} ; n, \mu(\lambda, n))=\lambda \log (1 / \lambda)^{-1 / a}+\int_{\lambda}^{1} \log (1 / x)^{-1 / a} d x .
\end{gathered}
$$

Thus, the ratio of these expressions is a lower bound on $V\left(\mathrm{MSB}_{\alpha}\right) / V(\mathrm{OMN})$.

The minimum of this lower bound for $\lambda \in(0,1)$ and $1 / a \in(0,1)$ exceeds 0.948 , completing the proof of the third claim.

LEMMA 4: Suppose that the $M_{i}$ are IID draws from a power law distribution with parameter a. Fix $\lambda \in(0,1)$ and let $\alpha=(1-\lambda)^{-1 / a}$, so that $\mathrm{MSB}_{\alpha}$ and OMN sell the impression to the brand advertiser with equal probability. Then $\frac{V_{P}\left(\mathrm{MSB}_{\alpha} ; n, \mu(\lambda, n)\right)}{V_{P}(\mathrm{OMN}) ; n, \mu(\lambda, n)}$ is decreasing in $n$.

\section{PROOF OF LEMMA 4:}

For this proof only, we adopt additional notation to indicate the number of bidders. We fix the match value distribution, let $E_{n}[\cdot]$ denote the expectation of its argument conditioned on $N=n$, and let $P_{n}(\cdot)$ denote the probability of the argument given $N=n$.

Fix $\lambda \in(0,1)$ and $a>1$, and let $\alpha=(1-\lambda)^{-1 / a}$. Note that Fact 1.2 implies that when $N=n+1$, the values $R_{i}=M_{(i)} / M_{(n+1)}$ for $i=1, \ldots, n$ are distributed as the order statistics of $n$ iid draws from a power law distribution with parameter $a$, and are independent from $M_{(n+1)}$. Thus,

$$
\begin{aligned}
V_{P}\left(\mathrm{MSB}_{\alpha} ; n+1\right) & =E_{n+1}\left[M_{(n+1)} R_{1} \mathbf{1}_{\frac{R_{1}}{R_{2}}>\alpha}\right] \\
& =E_{n+1}\left[M_{(n+1)}\right] E_{n+1}\left[R_{1} \mathbf{1}_{\frac{R_{1}}{R_{2}}>\alpha}\right] \\
& =E_{n+1}\left[M_{(n+1)}\right] V_{P}\left(\mathrm{MSB}_{\alpha} ; n\right) .
\end{aligned}
$$

The second line follows from the independence of $M_{(1)} / M_{(2)}$ from $M_{(n+1)}$ and the fact that $R_{1} / R_{2}=M_{(1)} / M_{(2)}$, while the final line follows from Fact 1.2. Thus, to prove the lemma, it suffices to show that for any $n \geq 2$,

$$
V_{P}(\mathrm{OMN} ; n+1) \geq E_{n+1}\left[M_{(n+1)}\right] V_{P}(\mathrm{OMN} ; n),
$$

We do this by considering an allocation rule $z$ such that

$$
V_{P}(z ; n+1)=E_{n+1}\left[M_{(n+1)}\right] V_{P}(\mathrm{OMN} ; n) .
$$

When $N=n+1$, this rule uses the ratio $R_{1}=M_{(1)} / M_{(n+1)}$ to determine how to allocate the impression: it goes to the top performance advertiser whenever $R_{1}$ exceeds $\mu(\lambda, n)$. Note that Fact 1.2 implies that $P_{n+1}\left(R_{1} \leq \mu(\lambda, n)\right)=P_{n}\left(M_{(1)} \leq\right.$ 
$\mu(\lambda, n))$, so this auction allocates the impression to the brand advertiser with the same probability $\lambda$ as under OMN. It follows that

$$
\begin{aligned}
V_{P}(\mathrm{OMN} ; n+1) & \geq E_{n+1}\left[M_{(n+1)} R_{1} \mathbf{1}_{R_{1}>\mu(\lambda, n)}\right] \\
& =E_{n+1}\left[M_{(n+1)}\right] E_{n+1}\left[R_{1} \mathbf{1}_{R_{1}>\mu(\lambda, n)}\right] \\
& =E_{n+1}\left[M_{(n+1)}\right] V_{P}(\mathrm{OMN} ; n)
\end{aligned}
$$

completing the proof.

LEMMA 5: Fix $n \geq 2$ and suppose that $P(N=n)=1$ and match values are drawn independently from a power law distribution with parameter a. If $\alpha=$ $(1-\lambda)^{-1 / a}$, then $\frac{V_{P}\left(\mathrm{MSB}_{\alpha} ; \mu(\lambda, n)\right)}{V_{P}(\mathrm{OMN} ; \mu(\lambda, n))}$ is decreasing in $\lambda$.

\section{PROOF OF LEMMA 5:}

We will prove the equivalent statement that the $\log$ of this ratio is decreasing. Lemmas 2 and 3 establish that $V_{P}(\mathrm{OMN})=E[C] \int_{\lambda}^{1} \mu(x, n) d x$ and $V_{P}\left(\mathrm{MSB}_{\alpha}\right)=$ $(1-\lambda)^{1-1 / a} E\left[X_{(1)}\right]$. It follows that

$$
\frac{d}{d \lambda} \log \left(V_{P}\left(\mathrm{MSB}_{\alpha}\right)\right)-\frac{d}{d \lambda} \log V_{P}(\mathrm{OMN})=\frac{-1}{1-\lambda}+\frac{\mu(\lambda, n)}{\int_{\lambda}^{1} \mu(x, n) d x} .
$$

Because $\mu(x, n)$ is increasing in $x, \int_{\lambda}^{1} \mu(x, n) d x>(1-\lambda) \mu(\lambda, n)$, proving that the expression above is negative.

LEMMA 6: Fix $\lambda \in(0,1)$ and $a>1$, and let $\alpha=(1-\lambda)^{-1 / a}$. Suppose that $N=n$, and that match values are drawn iid from a power law distribution with parameter $a$. Then the ratio $\frac{V\left(\mathrm{MSB}_{\alpha} ; \mu(\lambda, n), n\right)}{V(\mathrm{OMN} ; \mu(\lambda, n), n)}$ is decreasing in $n$.

PROOF OF LEMMA 6:

Note that for any allocation rules $A$ and $A^{\prime}$, it is possible to express the ratio of total value as a convex combination of the ratio of brand value and the ratio of performance value:

$$
\frac{V(A)}{V\left(A^{\prime}\right)}=\frac{V_{B}\left(A^{\prime}\right)}{V\left(A^{\prime}\right)} \cdot \frac{V_{B}(A)}{V_{B}\left(A^{\prime}\right)}+\frac{V_{P}\left(A^{\prime}\right)}{V\left(A^{\prime}\right)} \cdot \frac{V_{P}(A)}{V_{P}\left(A^{\prime}\right)},
$$

Fix $\lambda$ and $a$, and let $\alpha=(1-\lambda)^{-1 / a}$, so that the brand advertiser is equally likely to win the impression under $\mathrm{MSB}_{\alpha}$ and $\mathrm{OMN}$. Letting $A=\mathrm{MSB}_{\alpha}$ and $A^{\prime}=$ OMN above, we must show that for fixed $\lambda$ and $a$, the relative performance of MSB, as given in (11), is decreasing in $n$.

We know that $V_{B}\left(\mathrm{MSB}_{\alpha}\right)=V_{B}(\mathrm{OMN})$, and the first part of this Lemma establishes that the ratio $V_{P}\left(\mathrm{MSB}_{\alpha}\right) / V_{P}(\mathrm{OMN})$ is less than one and decreasing in $n$. Thus, it suffices to show that the ratio $V_{P}(\mathrm{OMN}) / V(\mathrm{OMN})$ is increasing in $n$ (fixing $\lambda$ and allowing $\mu=\mu(\lambda, n)$ to vary), or equivalently that $V_{P}(\mathrm{OMN} ; n, \mu(\lambda, n)) / V_{B}(\mathrm{OMN} ; n, \mu(\lambda, n))$ is increasing in $n$. 
Lemma 2 states that $V_{P}(\mathrm{OMN})=\int_{\lambda}^{1} \mu(x, n) d x$, and $V_{B}(\mathrm{OMN})=\lambda \mu(\lambda, n)$. It follows that

$$
\frac{V_{P}(\mathrm{OMN})}{V_{B}(\mathrm{OMN})}=\frac{1}{\lambda} \int_{\lambda}^{1} \frac{\mu(x, n)}{\mu(\lambda, n)} d x
$$

Suppose that $n^{\prime}>n$. We claim that $\frac{\mu\left(x, n^{\prime}\right)}{\mu(x, n)}$ is increasing in $x$. From this, it follows that

$$
\int_{\lambda}^{1} \frac{\mu\left(x, n^{\prime}\right)}{\mu\left(\lambda, n^{\prime}\right)} d x=\int_{\lambda}^{1} \frac{\mu\left(x, n^{\prime}\right)}{\mu(x, n)} \frac{\mu(x, n)}{\mu\left(\lambda, n^{\prime}\right)} d x \geq \int_{\lambda}^{1} \frac{\mu\left(\lambda, n^{\prime}\right)}{\mu(\lambda, n)} \frac{\mu(x, n)}{\mu\left(\lambda, n^{\prime}\right)} d x=\int_{\lambda}^{1} \frac{\mu(x, n)}{\mu(\lambda, n)} d x .
$$

All that remains is to prove our claim that $\frac{\mu\left(x, n^{\prime}\right)}{\mu(x, n)}$ is increasing in $x$. Note that

$$
\frac{d}{d x} \frac{\mu\left(x, n^{\prime}\right)}{\mu(x, n)}>0 \Leftrightarrow \frac{\frac{d}{d x} \mu\left(x, n^{\prime}\right)}{\mu\left(x, n^{\prime}\right)}-\frac{\frac{d}{d x} \mu(x, n)}{\mu(x, n)}>0 .
$$

Thus, it suffices to show that $\frac{d}{d x} \log (\mu(x, n))$ is increasing in $n$. We compute

$$
\frac{\frac{d}{d x} \mu(x, n)}{\mu(x, n)}=\frac{1}{a x} \frac{\frac{1}{n} x^{1 / n}}{\left(1-x^{1 / n}\right)}=\frac{1}{\operatorname{axn}\left(x^{-1 / n}-1\right)} .
$$

Making the substitution $z=1 / n$, we see that the above expression is increasing in $n$ if and only if $\left(x^{-z}-1\right) / z$ is increasing in $z$. But

$$
\begin{aligned}
\frac{d}{d z} \frac{x^{-z}-1}{z} & =\frac{1}{z^{2}}\left(-z \log (x) x^{-z}-\left(x^{-z}-1\right)\right) \\
& =\frac{1}{z^{2}}\left(x^{-z}\left(\log x^{-z}-1\right)+1\right)
\end{aligned}
$$

To see that this is non-negative, let $y=x^{-z}$. The minimum of $y(\log y-1)+1$ is at $y=1$, when the value of the expression is zero.

We now turn our attention to Corollary 1. Lemma 7 establishes that for any dominant strategy incentive compatible mechanism $(z, p)$, revenue from performance advertisers is at most $\left(1-a^{-1}\right) V_{P}(z)$, and that MSB auctions achieve this bound. If the publisher gets a fraction $\delta$ of the surplus from ads assigned to the brand advertiser, it follows that the revenue from the optimal mechanism is at most $\sup _{z} \delta V_{B}(z)+\left(1-a^{-1}\right) V_{P}(z)$; by Theorem 2, a suitably-chosen MSB auction gets at least $94.8 \%$ of this benchmark.

LEMMA 7: Suppose that $(z, p)$ is a dominant-strategy mechanism in which $x_{i}=$ 0 implies $p_{i}(x)=z_{i}(x)=0$. If $M_{i}$ is drawn from a power law distribution with parameter a, then $E\left[p_{i}(X)\right] \leq\left(1-a^{-1}\right) E\left[X_{i} z_{i}(X)\right]$, with equality if $(z, p)$ corresponds to an MSB auction.

\section{PROOF OF LEMMA 7:}


It is well-known that if the mechanism is dominant strategy incentive compatible for bidder $i$, then from this bidder's perspective, the mechanism makes a single take-it-or-leave-it offer. For any offer price $\hat{p}$ (which may depend arbitrarily on others' bids), we consider two cases:

1) $C=c>\hat{p}$. In this case, because $X_{i}>C$, bidder $i$ wins the impression, receives an expected value of $E\left[c M_{i}\right]=c /\left(1-a^{-1}\right)$, and pays $\hat{p}$, which is less than $\left(1-a^{-1}\right)$ times its expected value.

2) $C=c<\hat{p}$. In this case, bidder $i$ wins the impression whenever $M_{i}>\hat{p} / c$, and conditional on winning, has an expected value of $c(\hat{p} / c) \frac{a}{a-1}=\hat{p} /(1-$ $a^{-1}$ ) (by Fact 1.1). Bidder $i$ pays exactly $\hat{p}$ upon winning, implying that in this case, expected publisher revenues (from bidder $i$ ) are exactly $\left(1-a^{-1}\right.$ ) times expected total surplus (from bidder $i$ ).

Under an MSB auction, the threshold $\hat{p}=\alpha \max X_{-i}>\alpha C$ (since each $X_{j}>$ $C)$, so the first case above never occurs, and thus revenue and surplus from bidder $i$ are proportional. 